\title{
On the Validity of Haynes Rule for the Binding of Excitonic Complexes in Low Dimensions
}

\author{
(C) Jai Singh \\ Faculty of Science, Northern Territory university, \\ NT 0909 Darwin, Australia
}

Using a two-dimensional geometrical model and fractional dimension approach, it is found analytically that the ratio of the binding energy of a biexciton to that of an exciton is 0.228 in quantum wells and it is independent of the quantum well width. This agrees very well with the results in $\mathrm{GaAs}$ and $\mathrm{ZnSe}$ quantum wells, and $\mathrm{CuCl}$ crystals and large quantum dots. It is suggested that while Haynes rule may be valid for bulk, much higher ratios may be expected in lower dimensions.

1. Much interest has recently generated in studying the excitonic complexes like biexcitons and trions in narrow dimensions, because of their applications in quantum confined optoelectronic devices. In GaAs quantum well [1,2] and type II superlattices [3], the binding energy of biexcitons over $2 \mathrm{meV}$ has been observed, whereas in $\mathrm{ZnSe}$ quantum well [4] and $\mathrm{CuCl}$ single crystals [5-7] much higher binding energies have been measured. The first observation of biexcitons was made by Haynes [8] in crystalline silicon, and on the basis of his previous work on a neutral donor complex [9], Haynes used the ratio of the binding energy of a biexciton $\left(E_{b}^{x x}\right)$ to that of an exciton $\left(E_{b}^{x}\right)$ in silicon as 0.1. Eversince this ratio, commonly known as the Haynes Rule, has been assumed to be applicable for all solids, including quantum wells [10]. However, as biexcitons are observed more frequently in confined systems, a desirable question arises if there is a constant ratio for the binding energy of a biexciton to that of an exciton, which can be applied for both bulk and confined systems.

In this paper we have presented a brief account of determining the ratio of the binding energy of quasi-two-dimensional biexcitons to that of an exciton analytically [11] using the fractional dimension approach [12], and compared it with some of the known experimental results on quantum wells. As the Bohr radius of biexcitons is much larger than that of excitons, it may be expected that the effect of confinement will be more pronounced on biexcitons than on excitons. Therefore, while the Haynes rule may be regarded as applicable in bulk crystals, a higher ratio may be expected in lower dimensions.

2. In quantum wells of widths smaller than the biexcitonic diameter, a biexciton is confined into a $2 \mathrm{D}$ space. In this situation a planar square geometrical configuration [11] for the electrons and holes involved in the formation of a biexciton can be assumed. Assuming that the quantum well plane is parallel to the $x y$-plane, the $2 \mathrm{D}$ biexciton is free to move only in this plane. Transforming the Hamiltonian of such a geometrical structure into the six relative co-ordinates and a centre of mass coordinate, we get the biexciton
Hamiltonian as [11]

$$
\begin{gathered}
H_{x x}=-\frac{\hbar^{2}}{2 M} \nabla_{R}^{2}-\frac{\hbar^{2}}{2}\left(\frac{2}{\mu_{\mathrm{eh}}} \nabla_{\mathrm{eh}}^{2}+\frac{1}{\mu_{\mathrm{ee}}} \nabla_{\mathrm{ee}}^{2}\right. \\
\left.+\frac{1}{\mu_{\mathrm{hh}}} \nabla_{\mathrm{hh}}^{2}\right)+V
\end{gathered}
$$

where $\quad M=2\left(m_{\mathrm{e}}^{*}+m_{\mathrm{h}}^{*}\right), \quad 1 / \mu_{\mathrm{eh}}=1 / m_{\mathrm{e}}^{*}+1 / m_{\mathrm{h}}^{*}$, $1 / \mu_{\mathrm{ee}}=1 / m_{\mathrm{e}}^{*}$ and $1 / \mu_{\mathrm{hh}}=2 / m_{\mathrm{h}}^{*}, \quad \nabla_{\mathrm{eh}}^{2}, \quad \nabla_{\mathrm{ee}}^{2}$ and $\nabla_{\mathrm{hh}}^{2}$ are the Laplacians with respect to the relative co-ordinates between electron and hole, electron and electron, and hole and hole, respectively, and $\nabla_{R}^{2}$ is that with respect to the centre of mass co-ordinate. $V$ is the Coulomb potential of interaction among the electrons and holes of the biexciton. Applying another co-ordinate transformation to the relative co-ordinates that ensures a square structure of the $2 \mathrm{D}$ biexciton, the Hamiltonian (1) reduces into [11]

$$
H_{x x}=-\frac{\hbar^{2}}{2 \mu_{x x}} \nabla_{r}^{2}-\frac{e^{2}}{\varepsilon_{e e} r},
$$

where $\mu_{x x}=\frac{2}{3} \mu_{\mathrm{eh}}, \varepsilon_{x x}=(\sqrt{2} /(4-\sqrt{2})) \varepsilon$, and $\varepsilon$ is the dielectric constant of the material. The kinetic energy operator of the centre of mass motion is excluded from the Hamiltonian (2). The energy eigenvalue of the Hamiltonian (2) can be obtained be applying the fractional dimension approach [12] in solving the following Schrödinger equation

$$
H_{x x} \psi_{x x, n}(r)=\left(E_{x x, n}-2 E_{g}\right) \psi_{x x, n}(r),
$$

where $E_{x x, n}$ and $\psi_{x x, n}$ are the energy eigenvalue and eigenfunction of a biexciton in its internal energy state $n$, respectively, and $E_{g}$ is the energy gap of the material. We thus obtain

$$
E_{x x, n}=2 E_{g}-\frac{E_{B}^{\prime}}{(n+((\alpha-3) / 2))^{2}}, \quad \alpha=2,
$$

and the corresponding biexciton radius $a_{x x, n}$ as

$$
a_{x x, n}=\left(n+\frac{\alpha-3}{2}\right)^{2} a_{x}^{\prime}
$$

where

$$
E_{B}^{\prime}=\frac{\mu_{x x}}{\varepsilon_{x x}^{2} m_{\mathrm{e}}} R_{H}
$$


and

$$
a_{x}^{\prime}=\frac{m_{\mathrm{e}}}{\varepsilon_{x x} \mu_{x x}} a_{H} .
$$

$R_{H}$ and $a_{H}$ are the Rydberg constant and Bohr radius, respectively, and $m_{\mathrm{e}}$ is the free electron mass.

Using the definition of the biexciton binding energy as $E_{b}^{x x}=2 E_{x, n}-E_{x x, n}$, where $E_{x, n}$ is the energy eigenvalue of an exciton state, we get the exciton binding energy as

$$
E_{b}^{x x}(n)=\left(\frac{(4-\sqrt{2})^{2}}{3}-2\right) E_{b}^{x}(n)
$$

where $E_{b}^{x}(n)=E_{g}-E_{x, n}$, is the binding energy of a $2 \mathrm{D}$ exciton. From (8) one gets the ratio, $E_{b}^{x x} / E_{b}^{x}=0.228$, which does not depend on the biexciton state principal quantum number $n$, effective masses of the charge carriers and quantum well width. The ratio is also more than twice the ratio obtained by Haynes rule.

Using Eq. (5), we also find the ratio of the radius of biexction to exciton as

$$
\frac{a_{x x, n}}{a_{x, n}}=\frac{3(4-\sqrt{2})}{2 \sqrt{2}}=2.74,
$$

which is also independent of $n$ and therefore the ratio is same for the Bohr radius of biexciton to that of exciton. The ratio (9) suggests that the biexction Bohr radius can be more than twice as large as the exciton Bohr radius.

3. Using a simple but realistic model structure of $2 \mathrm{D}$ biexcitons in quantum wells, we have obtained analytically $E_{b}^{x x} / E_{b}^{x}=0.228$ for $2 \mathrm{D}$ biexcitons. The questions now arise (i) Do biexcitons in all quantum well materials have the same ratio? (ii) Although the results derived here are strictly applicable for the two dimensional case, can this ratio be applied in dimensions lower than 2D (quantum wires, quantum dots etc.)? (iii) Should it really be independent of the quantum well width? We would like to address these questions below.

Material independence of $E_{b}^{x x} / E_{b}^{x}$. Biexcitons have been observed in many quantum wells, but they have been studied more systematically only in GaAs quantum wells [2]. In GaAs quantum wells the measured ration is 0.2 , and the binding energy of quasi-2D excitons in GaAs quantum wells of width $80 \AA$ is known [2] to be $10 \mathrm{meV}$ which gives a binding energy of $2 \mathrm{meV}$ for biexcitons. In $\mathrm{ZnSe}$ single quantum well of width $75 \AA$ [4] the measured binding energy of biexciton is $5 \mathrm{meV}$ and the exction binding energy is $20-25 \mathrm{meV}$, which gives a ratio of $0.2-0.25$.

In the bulk crystals of $\mathrm{CuCl}$ the binding energy of $\Gamma_{1}$-biexction, the lowest bound state of two $Z_{3}$ excitons, has a binding energy of $32 \mathrm{meV}[5]$ and the exciton binding energy is $150-190 \mathrm{meV}$, which gives a ratio of $0.17-0.21$. To the author's knowledge, this is the only example of such a large binding energy of biexcitons in a bulk. The ratio in Eq. (8) is clearly in agreement with the above examples which suggest that the ratio is independent of the material.
However, this is not true for trions (a free charge carrier bound to an exciton), where the ratio $E_{b}^{x x} / E_{b}^{x}$ depends on $\sigma=m_{\mathrm{e}}^{r} / m_{\mathrm{h}}^{*}[13,14]$, and hence it is different for different materials. For a negatively charged trion $(\mathrm{e}-\mathrm{h}-\mathrm{e})$, the binding energy is obtained as

$$
E_{b}^{x-}=\left[\frac{9(1+\sigma)}{2(3+2 \sigma)}-1\right] E_{b}^{x}
$$

and hence $E_{b}^{x-} / E_{b}^{x}$ depends on $\sigma$. Using Eq. (10) we get a ratio of $E_{b}^{x-}$ to the energy of a neutral donor as a function of $\sigma$ that agrees reasonably well with the variational calculation [14]. Such a difference between $E_{b}^{x x} / E_{b}^{x}$ and $E_{b}^{x-} / E_{b}^{x}$ may be attributed to the difference in the structure of biexcitons and trions.

E f f e c t of Confine ment. As the biexciton Bohr radius is more than twice larger than the exciton Bohr radius [Eq. (9)], one expects that the effect of confinement would be more pronounced on the binding of biexcitons than on excitons. This means that the ratio $E_{b}^{x x} / E_{b}^{x}$ is expected to be larger in narrow dimensions such that it is least in bulk and highest in 1D and 0D. To our knowledge there are only two examples, $\mathrm{GaAs}$ and $\mathrm{CuCl}$, which may be used here. In GaAs quantum wires the ratio obtained from a very sophisticated variational calculation [15] suggests that $E_{b}^{x x} / E_{b}^{x}=0.6$ and it is nearly independent of the length and width of quantum wires. The experimental results [2] in GaAs quantum wells show that $E_{b}^{x x} / E_{b}^{x}=0.2$ and it is independent of the quantum well width, which is also supported by the ratio obtained in Eq. (8).

In $\mathrm{CuCl}$ quantum dots of sizes more than $50 \AA$, no effect of confinement on biexcitons and excitons is observed [16]. This is because the $1 S$ exciton Bohr radius in $\mathrm{CuCl}$ is $6.8 \AA[13]$ and the corresponding biexciton Bohr radius is $15 \AA$ [16], and the effect of confinement is not found to be significant in quantum dot sizes much bigger than exciton and biexciton Bohr radii [17]. However, more experimental data are needed on the binding energy of excitons and biexcitons as a function of the size of confinement to address the issue any further. Nevertheless, it is interesting to see from the above data $[16,18]$ that the ratio of biexciton to exciton Bohr radii is 2.2 in $\mathrm{CuCl}$, which is comparable with 2.7 obtained in Eq. (9).

I am indebted to Professor T. Itoh for supplying his results on $\mathrm{CuCl}$ and to Dr. D. Birkedal for many useful discussions. A part of the work was done at the Mikroelectronik Centret, Danish Techical Untiversity, and I would like to thank Professor J. Hvam for the hospitality.

\section{References}

[1] R.C. Miller, D.A. Kleinman, A.C. Gossard, O. Munteanu. Phys. Rev. B25, 6545 (1982).

[2] D. Birkedal, J. Singh, V.G. Lyssenko, J. Erland, J.M. Hvam. Phys. Rev. Lett. 76, 672 (1996).

[3] M. Nakayama, K. Suyama, H. Nishimura. Fourth Intl. Meeting Optics of excitons in confined systems. Cortona, Italy (28-31 Aug. 1995). 
[4] V. Kozlov, P. Kelkar, A.V. Nurmikko. Phys. Rev. B53, 10837 (1996).

[5] R. Shimano, M. Kuwata-Gonokami. Phys. Rev. Lett. 72, 530 (1994).

[6] K. Saito, N. Nagasawa. Solid State Commun. 94, 33 (1995).

[7] M. Hasuo, N. Nagasawa. Phys. Rev. Lett. 70, 1303 (1993).

[8] J.R. Haynes. Phys. Rev. Lett. 17, 860 (1966).

[9] J.R. Haynes. Phys. Rev. Lett. 4, 361 (1960).

[10] D.A. Kleinman. Phys. Rev. B28, 871 (1983).

[11] J. Singh, D. Birkedal, V.G. Lyssenko, J.M. Hvam. Phys. Rev. B53, 15909 (1996).

[12] X.F. He. Phys. Rev. B43, 2063 (1991); H. Mathieu, P. Lefebvre, P. Christol. Phys. Rev. B48, 17308 (1993).

[13] J. Singh. Appl. Opt., in press (1996).

[14] B. Stebe, A. Ainane. Superlatt. Microstruct. 5, 545 (1989).

[15] F. Madarasz. Phys. Rev. B49, 13528 (1994).

[16] K. Yamanaka, K. Edamatsu, T. Itoh. J. Lumin. (DPC'97), in press.

[17] M. Hasuo, N. Nagasawa, T. Itoh, A. Mysyrowicz. J. Lumin. 60 \& 61, 758 (1994).

[18] Y. Mimura et al. J. Lumin. 66 \& 67, 401 (1996). 